\title{
Catalytically Important Residues in E. coli 1-Deoxy-D-Xylulose 5-Phosphate Synthase
}

\author{
Jordi Querol-Audí, Albert Boronat, Josep J. Centelles, Santiago Imperial \\ Department of Biochemistry and Molecular Biology, School of Biology, University of Barcelona, Barcelona, \\ Spain \\ Email: simperial@ub.edu
}

Received March 2014

\section{Abstract}

1-deoxy-D-xylulose 5-phosphate synthase (DXS) catalyzes the initial step of the 2-C-methyl-Derythritol 4-phosphate (MEP) pathway consisting in the condensation of (hydroxiethyl)thiamin derived from pyruvate with D-glyceraldehyde 3-phosphate (GAP) to yield 1-deoxy-D-xylulose 5-phosphate (DXP). The role of the conserved residues H49, E370, D427 and H431 of E. coli DXS was examined by site-directed mutagenesis and kinetic analysis of the purified recombinant enzyme mutants. Mutants at position $\mathrm{H} 49$ showed a severe reduction in their specific activities with a decrease of the $\boldsymbol{k}_{c a t} / K_{M}$ ratio by two orders of magnitude lower than the wild-type DXS. According to available structural data residue $\mathrm{H} 49$ is perfectly positioned to abstract a proton from the donor substrate. Mutations in DXS E370 showed that this residue is also essential for catalytic activity. Three-dimensional structure supports its involvement in cofactor deprotonation, the first step in enzymatic thiamin catalysis. Results obtained with $\mathrm{H} 431$ mutant enzymes indicate that this residue plays a role contributing to transition state stabilization. Finally, mutants at position D427 also showed a severe specific activity decrease with a reduction of the $k_{c a t} / K_{M}$ ratio. A role in binding the substrate and selecting the stereoisomer is proposed for D427.

\section{Keywords}

Active Site, 1-Deoxy-D-Xylulose 5-Phosphate Synthase, Isoprenoid Biosynthesis, Kinetic Parameters, MEP Pathway, Methylerythritol Phosphate, Mutagenesis

\section{Introduction}

Isoprenoids, the most diverse group of natural products with thousands of compounds identified [1] derive from two common C5 units: isopentenyl diphosphate (IPP) and its isomer dimethylallyl diphosphate (DMAPP). In most eubacteria IPP and DMAPP biosynthesis proceed exclusively through the methylerythritol 4-phosphate (MEP) pathway [2] [3]. In the first reaction pyruvate and D-glyceraldehyde 3-phosphate (GAP) are condensed to yield deoxyxylulose 5-phosphate (DXP). This involves the decarboxylation of pyruvate and the transfer of the resulting C2 unit to the aldehyde group of GAP (Figure 1) in a reaction dependent on thiamin diphosphate (TDP).

This reaction is catalyzed by DXP synthase (DXS) [4]-[6], an enzyme belonging to the superfamily of TDPdependent enzymes including transketolases and the E1 subunit of pyruvate dehydrogenase. Then, DXP is con- 


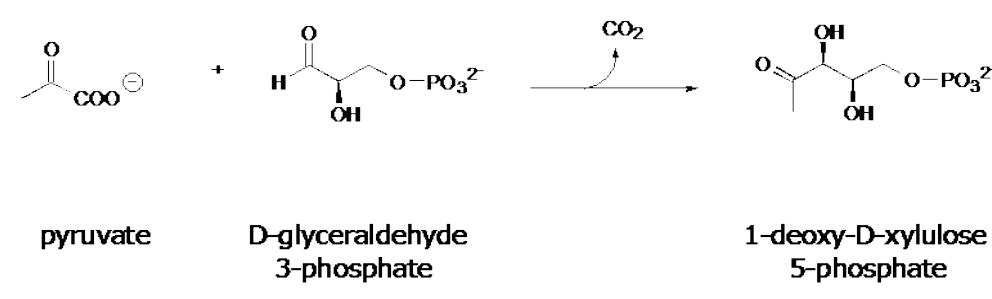

Figure 1. Reaction catalyzed by deoxyxylulose 5-phosphate synthase (DXS).

verted by intramolecular rearrangement and reduction into 2-C-methyl-D-erythritol 4-phosphate (MEP) in a reaction catalyzed by DXP reductoisomerase (DXR) [7]. Since DXP is also the substrate for the synthesis of vitamins B1 (thiamin) and B6 (pyridoxol) [8] [9], MEP is the first specific intermediate of the pathway and it is accepted to name it as MEP pathway. Many pathogenic microorganisms, such as M. tuberculosis [10] and the malaria parasite $P$. falciparum [11], synthesize IPP and DMAPP through the MEP pathway. Given the essential role of isoprenoids in these organisms, and the fact that the MEP pathway is absent in mammals, where these precursors are exclusively synthesized via the mevalonate pathway, all the enzymes participating in the MEP pathway are potential targets for the design of inhibitors that could be used as antibiotic or antimalarial drugs [10] [11]. In this context, DXS is one of the most attractive targets, because of its regulatory role in the biosynthesis of isoprenoids in various systems [12] [13], indicating that this enzyme catalyzes a rate-limiting step in the MEP pathway. Identification of catalytically essential amino acid residues is necessary in order to design effective drugs. In the present work we analyzed the role of other conserved residues in DXS activity by site-directed mutagenesis. Nine recombinant proteins (wild-type, H49Q, H49A, E370Q, E370A, D427N, D427A, H431Q and H431A) were purified to apparent homogeneity using $\mathrm{Ni}^{2+}$-affinity chromatography and kinetic analysis of the mutant enzymes were performed in order to examine their catalytic role in E. coli DXS.

\section{Materials and Methods}

\subsection{Materials}

Materials from commercial sources included NADPH, DL-glyceraldehyde 3-phosphate (DL-GAP) and TDP from Sigma. Hi-Trap Chelating columns were from GE Healthcare. The protease inhibitors Pefabloc SC Plus and the protease inhibitor cocktail Complete EDTA-free were obtained from Roche. All other reagents were of analytical grade. Recombinant E. coli DXR was obtained as described in [14].

\subsection{Construction of the E. coli DXS Expression Vector pET-23-DXS}

The coding region of the E. coli DXS, cloned into the expression vector pT7-7 [15], was amplified by PCR using Pfu DNA polymerase and primers T7 (5'-TAATACGACTCACTATAGG-3') and pET-23-XhoI

(5'-CGCTCGAGTCCTGCCAGCCAGGCCTTGATTTTGGC-3’). After digestion with NdeI and XhoI, the amplified DNA fragment was ligated into the same sites of the expression vector pET-23(b). Strain DH5 $\alpha$ (Promega) was used as the recipient during this transformation. Positive clones were identified by DNA sequencing. The resulting plasmid, pET-23-DXS, produces a C-terminal histidine-tagged enzyme (DXS-cHis).

\subsection{Site-Directed Mutagenesis}

Point mutations H49Q, H49A, E370Q, E370A, D427N, D427A, H431Q and H431A were introduced into the E. coli DXS coding sequence by site-directed mutagenesis using the QuikChange Site-Directed Mutagenesis Kit (Stratagene) as described [15]. Primers used for introducing these point mutations are summarized in Table $\mathbf{1 .}$ The underlined letters represent the substituted nucleotides. Presence of the desired mutations and absence of other mutations that could be introduced during PCR were verified by DNA sequencing.

\subsection{Overexpression and Purification of Cloned DXS Proteins}

BL21 (DE3) pLysS cells carrying pET-23-DXS or the corresponding mutants were grown in LB medium supplemented with $100 \mu \mathrm{g} / \mathrm{ml}$ ampicillin and $34 \mu \mathrm{g} / \mathrm{ml}$ chloramphenicol at $22^{\circ} \mathrm{C}$ to an absorbance at $600 \mathrm{~nm}$ of $0.3-$ 0.4 and then induced with $0.3 \mathrm{mM}$ isopropyl $\beta$-D-thiogalactoside for 18 - $20 \mathrm{~h}$. Bacterial cells were recovered by 
Table 1. Primers used in this study for site-directed mutagenesis ${ }^{\mathrm{a}}$.

\begin{tabular}{|c|c|}
\hline name & Sequence \\
\hline H49Q & 5'-CCAGCGGGCAGTTCGCCTCC-3' \\
\hline H49A & 5'-TCCAGCGGGGCCTTCGCCTC-3' \\
\hline E370Q & 5'-GCAATTGCCCAGCAACACGCGG-3' \\
\hline E370A & 5'-GCAATTGCCGCGCAACACGCG-3' \\
\hline D427N & 5'-TGTTGGTGCTAACGGTCAAACCC-3' \\
\hline D427A & 5'-GTTGGTGCTGCCGGTCAAACCC-3' \\
\hline H431Q & 5'-GGTCAAACCCAGCAGGGTGCTT-3' \\
\hline H431A & 5'-GGTCAAACCGCTCAGGGTGCTT-3' \\
\hline
\end{tabular}

${ }^{\mathrm{a}}$ The underlined letters represent the substituted nucleotides.

centrifugation and the cell pellet was resuspended in Buffer 1 (40 mM Tris-HCl, pH 8.5, $100 \mathrm{mM} \mathrm{NaCl}, 10 \mathrm{mM}$ imidazole, $1 \mathrm{mM} \mathrm{MgCl}$, $5 \mathrm{mM}$ 2-mercaptoethanol) containing $1 \mathrm{mM}$ TDP, lysozyme (1 mg/ml), Pefabloc SC Plus $(1 \mathrm{mM})$ and one tablet of protease inhibitor cocktail Complete EDTA-free. After incubation at $4^{\circ} \mathrm{C}$ for 30 min and brief sonication ( 3 pulses, $30 \mathrm{w}, 20 \mathrm{~s}$ ), lysate was centrifuged at 12,000 $\times \mathrm{g}$ for $45 \mathrm{~min}$ at $4^{\circ} \mathrm{C}$. Recombinant DXSs were purified by $\mathrm{Ni}^{2+}$ affinity chromatography (1ml Hi-Trap Chelating column, GE Healthcare) with a linear gradient of 10 to $500 \mathrm{mM}$ imidazole. Fractions containing DXS were pooled, dialyzed by ultrafiltration (Ultrafree- 0.5 and Ultrafree-4 centrifugal filter Biomax-5K, Millipore) against $50 \mathrm{mM}$ glycylglycine buffer (pH 7.8), $1 \mathrm{mM} \mathrm{MgCl}, 1 \mathrm{mM}$ TDP, $5 \mathrm{mM} 2$-mercaptoethanol and stored at $-20^{\circ} \mathrm{C}$ in $50 \%$ glycerol. Purified fractions were analyzed by SDS-PAGE. After dialyzing the samples against (1) glycylglycine buffer $10 \mathrm{mM}$, pH 7.8, 1 mM DTT, 50 mM EDTA and (2) glycylglycine buffer 10mM, pH 7.8, 1 mM DTT, protein concentration was determined by measuring the absorbance at $280 \mathrm{~nm}$ using an $\mathrm{Abs} 1 \%=6.25$.

\subsection{DXS Activity Measurements and Determination of Kinetic Parameters}

Specific activity for wild-type and mutant DXS proteins was monitored by a coupled system using purified $E$. coli DXR [16]. All kinetic measurements were made at $37^{\circ} \mathrm{C}$ in triplicate. The standard reaction mixture consisted in $50 \mathrm{mM}$ glycylglycine buffer $\mathrm{pH}$ 7.8, $1 \mathrm{mM}$ TDP, $1 \mathrm{mM} \mathrm{MgCl}_{2}, 0.05 \mathrm{mM} \mathrm{MnCl}_{2}, 1 \mathrm{mM}$ DTT, $0.15 \mathrm{mM}$ NADPH, $1 \mathrm{mM}$ pyruvate, $50 \mu \mathrm{g}$ DXR and variable amounts $(10 \mu \mathrm{g}$ to $160 \mu \mathrm{g})$ of DXS or the corresponding mutants in a final volume of $1 \mathrm{ml}$. Mixtures were incubated at $37^{\circ} \mathrm{C}$ for $10 \mathrm{~min}$ and the reaction was initiated by adding $1 \mathrm{mM}$ DL-GAP. Reactions were conducted at $37^{\circ} \mathrm{C}$ and initial velocities were monitored at $340 \mathrm{~nm}$ due to the oxidation of NADPH in a UNICAM UV-3 spectrophotometer. Specific activities were determined from the slope of a plot of initial velocities versus the enzyme amount.

Steady-state kinetic parameters for the wild-type and mutant enzymes were determined at saturating concentrations of the second substrate, $0.05 \mathrm{mM}$ to $1.6 \mathrm{mM}$ pyruvate and $25 \mu \mathrm{M}$ to $800 \mu \mathrm{M}$ DL-GAP, by measuring initial rates. $K_{M}$ values for pyruvate and GAP were calculated by fitting the data to the Michaelis-Menten equation using the program GRAFIT. For mutants H49Q, H49A, D427N, D427A and H431Q, $k_{\text {cat }} / K_{M}$ values for pyruvate were calculated from initial velocities at unsaturating concentrations of this substrate $(30 \mu \mathrm{M})$.

\section{Results and Discussion}

\subsection{Expression of DXS-cHis Mutants in E. coli}

The gene encoding E. coli DXS was cloned into the expression vector pET-23. Mutations at positions H49, E370, D427 and H431 were introduced by site-directed mutagenesis. DNA sequencing confirmed that undesired mutations were not introduced during the course of mutagenesis. Over expression of the wild-type and mutant enzymes was carried out in E. coli BL21 (DE3) pLysS. Purification of the recombinant proteins, containing a His-tag at the C-terminus, was performed in a single chromatographic step (Hi-Trap Chelating, GE Healthcare). Wild-type and mutant DXS proteins were obtained in similar amounts ( $\geq 10 \mathrm{mg}$ of pure DXS/litre) and purified $(>95 \%)$ as shown by SDS-PAGE and Coomassie-blue staining (Figure 2(a)). The enzyme purity was comparable for the wild-type and the mutant proteins. 


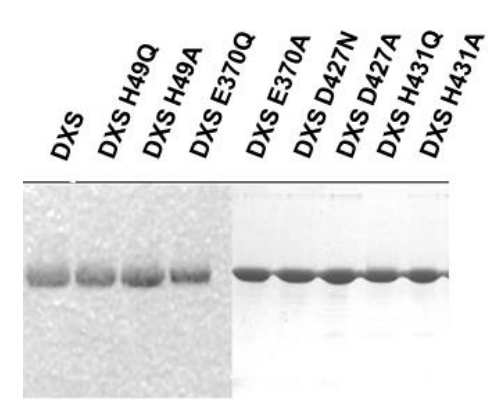

(a)

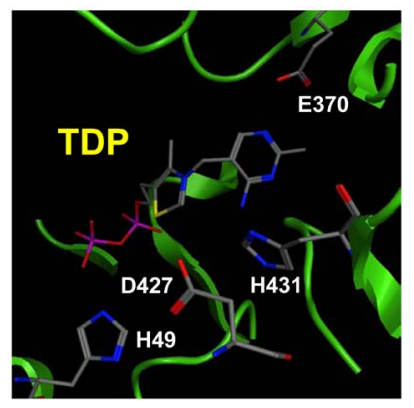

(b)

Figure 2. (a) SDS-PAGE analysis of purified E. coli DXS mutants. Purified DXS mutants obtained by using a Hi-Trap Chelating column were analyzed by SDS-PAGE. Proteins were stained with Coomassie brilliant blue R-250. Purified wild type and mutant proteins are indicated in the top of the gel; (b) Pose of the mutated residues and the coenzyme TDP at the catalytic site of E. coli DXS based on structural data reported at [17].

\subsection{Kinetic Studies}

E370Q and E370A mutations resulted in a completely inactive DXS and the H431A mutant showed only a moderate decrease in its specific activity compared to the wild-type enzyme $(0.84$ and $1.46 \mu \mathrm{mol}$ pyruvate/min $\mu \mathrm{g}$, respectively). The greatest decrease in activity levels were observed with the H49Q, H49A, D427N and D427A mutants which showed only $2 \%, 6 \%, 3 \%$ and $10 \%$ of the activity of the wild-type DXS. H431Q also exhibited a reduced catalytic activity (4\% of the wild-type) while H431A mutant retained about $57 \%$ activity.

For mutants at positions H49, D427 and H431Q $k_{c a t}$ values were obtained from the plot of initial velocities versus the enzyme amount. $k_{c a t} / K_{M}$ values for pyruvate were determined by measuring the initial velocities at subsaturating concentrations of pyruvate (Table 2).

We previously reported that mutation of residue $\mathrm{H} 49$ by glutamine in $E$. coli DXS resulted in a mutant enzyme with undetectable activity [15]. Here we have analyzed the effect of H49Q and H49A point mutations by measuring their kinetic parameters $k_{c a t}$ and $k_{c a t} / K_{M}$. Both mutations result in severally impaired catalytic activities. $k_{c a d} / K_{M}$ values for mutant H49A was two orders of magnitude lower than the wild-type enzyme. No kinetic parameters could be determined for mutant $\mathrm{H} 49 \mathrm{Q}$ due to its reduced specific activity. The H431A mutant retained $57 \%$ specific activity but increased $K_{M}$ for pyruvate (one order of magnitude compared to the wild-type). No significant change in the $K_{M}$ for GAP was observed for this mutant. When residue H431 was replaced by glutamine, $k_{\text {cat }}$ was reduced by two orders of magnitude. Kinetic analysis of the purified D427N and D427A mutants showed reduced $k_{\text {cat }}$ values ( $3.3 \mathrm{~min}^{-1}$ and $10 \mathrm{~min}^{-1}$ respectively, compared to the value of $100.3 \mathrm{~min}^{-1}$ for the wild-type enzyme) (Table 2).

Site-directed mutagenesis was used to confirm the essential role of selected residues for E. coli DXS activity. Kinetic parameters of the mutant enzymes H49Q and H49A obtained in this work corroborate that this residue is essential for DXS activity. The structure of the catalytic center of E. coli DXS [17] confirms that residue H49 is perfectly positioned to abstract a proton from the donor substrate.

Kinetic analysis of the purified DXS mutant enzymes at the corresponding positions (E370, D427 and H431) were carried out to establish the role of these conserved residues for E. coli DXS catalytic activity. Residue E418, equivalent to E370 in DXS, has been reported to be essential for TK catalytic activity since its implication in cofactor deprotonation, the first step in enzymatic thiamin catalysis [18]. Analysis of mutations in DXS E370 demonstrated that this residue is also essential for catalytic activity. E. coli DXS 3D-structure [17] shows a direct hydrogen bond to the pyrimidine of thiamin supporting the catalytic role suggested by Wikner et al. [19].

Site-directed mutagenesis studies of residue $\mathrm{H} 481$ in yeast transketolase indicate that this residue contributes to transition state stabilization but is not absolutely required for catalysis [19]. When the E. coli DXS histidine residue at position 431 was replaced by glutamine, a mutant DXS with a severe reduction in enzyme activity ( $4 \%$ of the wild-type) was obtained. In contrast, moderated effects in terms of specific activity and $k_{\text {cat }}$ were observed when $\mathrm{H} 431$ was replaced by alanine. Only $K_{M}$ for pyruvate was increased one order of magnitude (Table 2). Similar results have been obtained for mutants at residue $\mathrm{H} 481$ in yeast transketolase [19] or the correspond- 
Table 2. Comparison of kinetic parameters of wild-type (DXSwt) and mutant DXS enzymes.

\begin{tabular}{ccccc}
\hline Enzyme & $K_{\text {Mpyr }}(\mu \mathrm{M})$ & $K_{M G A P}(\mu \mathrm{M})$ & $k_{c a t}\left(\mathrm{~min}^{-1}\right)$ & $k_{\text {cat }} / K_{\text {Mpyr }}\left(\mathrm{min}^{-1} \cdot \mu \mathrm{M}^{-1}\right)$ \\
\hline DXSwt & $134 \pm 8$ & $41 \pm 6$ & 100.3 & 0.7480 \\
H49Q & n.d. & n.d. & n.d. & n.d. \\
H49A & n.d. & n.d. & 6.6 & 0.0091 \\
D427N & n.d. & n.d. & 3.3 & 0.0022 \\
D427A & n.d. & n.d. & 10.0 & 0.0095 \\
H431Q & n.d. & n.d. & 4.4 & 0.1600 \\
H431A & 1623 \pm 81 & $62 \pm 6$ & 57.4 & 0.0350 \\
E370Q & n.d. & n.d. & n.d. & n.d. \\
E370A & n.d. & n.d. & n.d. & n.d. \\
\hline
\end{tabular}

ing residue in human transketolase Q428 [20]. Site-directed mutagenesis studies of H481 in yeast transketolase suggested that this residue contributes to transition state stabilization but is not required for catalysis [20]. Our results would indicate that TK H481 and DXS H431 are conserved equivalent residues playing similar roles. The fact that H431Q mutant shows a markedly reduced specific activity could be explained by considering that the increase in side chain size may result in steric clashes with the substrate.

Substitution of aspartic acid residue at position 427 by glutamine or alanine yielded a DXS with highly decreased specific activity with $k_{c a t} / K_{M}$ ratio reduced by two orders of magnitude. The hydrogen at the side chain of the corresponding residue, D477, in yeast TK is proposed to be the determinant of the enantioselectivity of the enzyme [21].

In this study we describe the active site architecture of 1-deoxy-D-xylulose 5-phosphate synthase, a target for antimicrobial agents, herbicides, and antimalarial drugs [22] [23]. A role in catalysis has been assigned to conserved amino acid residues and a model of the catalytic site has been generated which can be of great interest to predict drug-enzyme interactions.

\section{References}

[1] Chappell, J. (2002) The Genetics and Molecular Genetics of Terpene and Sterol Origami. Current Opinion in Plant Biology, 5, 151-157. http://dx.doi.org/10.1016/S1369-5266(02)00241-8

[2] Zhao, L.S., Chang, W.C., Xiao, Y.L., Liu, H.W. and Liu, P.H. (2013) Methylerythritol Phosphate Pathway of Isoprenoid Biosynthesis. Annual Review of Biochemistry, 82, 497-530. http://dx.doi.org/10.1146/annurev-biochem-052010-100934

[3] Rohmer, M., Knani, M., Simonin, P., Sutter, B. and Sham, H. (1993) Isoprenoid Biosynthesis in Bacteria: A Novel Pathway for Early Steps Leading to Isopentenyl Diphosphate. The Biochemical Journal, 295, 517-524.

[4] Eubanks, L.M. and Poulter C.D. (2003) Rhodobacter capsulatus 1-Deoxy-D-Xylulose 5-Phosphate Synthase: SteadyState Kinetics and Substrate Binding. Biochemistry, 42, 1140-1149. http://dx.doi.org/10.1021/bi0205303

[5] Sprenger, G.A., Schörken, U., Wiegert, T., Grolle, S., De Graaf, A., Taylor, S., Begley, T., Bringer-Meyer, S. and Sahm, H. (1997) Identification of a Novel Thiamin-Dependent Synthase in Escherichia coli Required for the Formation of the 1-Deoxy-D-Xylulose 5-Phosphate Precursor to Isoprenoids, Thiamin, and Pyridoxol. Proceedings of the National Academy of Sciences of the USA, 94, 12857-12862. http://dx.doi.org/10.1073/pnas.94.24.12857

[6] Lois, L.M., Campos, N., Putra, S.R., Danielsen, K., Rohmer, M. and Boronat, A. (1998) Cloning and Characterization of a Gene from Escherichia coli Encoding a Transketolase-Like Enzyme That Catalyzes the Synthesis of D-1-Deoxyxylulose 5-Phosphate, a Common Precursor for Isoprenoid, Thiamin, and Pyridoxol Biosynthesis. Proceedings of the National Academy of Sciences of the USA, 95, 2105-2110. http://dx.doi.org/10.1073/pnas.95.5.2105

[7] Proteau, P.J. (2004) 1-Deoxy-D-Xylulose 5-Phosphate Reductoisomerase: An Overview. Bioorganic Chemistry, 32, 483-493. http://dx.doi.org/10.1016/j.bioorg.2004.08.004

[8] Himmeldirk, K., Kennedy, I.A., Hill, R.E., Sayer, B.G. and Spenser, I.D. (1996) Biosynthesis of Vitamins B1 and B6 in Escherichia coli: Concurrent Incorporation of 1-Deoxy-D-Xylulose into Thiamin (B1) and Pyridoxol (B6). Chemical Communications, 10, 1187-1188. http://dx.doi.org/10.1039/cc9960001187 
[9] Mukherjee, T., Hanes, J., Tews, I., Ealick, S.E. and Begley, T.P. (2011) Pyridoxal Phosphate: Biosynthesis and Catabolism. Biochimica et Biophysica Acta, 1814, 1585-1596. http://dx.doi.org/10.1016/j.bbapap.2011.06.018

[10] Obiol-Pardo, C., Rubio-Martinez, J. and Imperial, S. (2011) The Methylerythritol Phosphate (MEP) Pathway for Isoprenoid Biosynthesis as a Target for the Development of New Drugs against Tuberculosis. Current Medicinal Chemistry, 18, 1325-1338. http://dx.doi.org/10.2174/092986711795029582

[11] Wiesner, J. and Jomaa, H. (2007) Isoprenoid Biosynthesis of the Apicoplast as Drug Target. Current Drug Targets, 8, 3-13. http://dx.doi.org/10.2174/138945007779315551

[12] Lois, L.M., Rodríguez-Concepción, M., Gallego, F., Campos, N. and Boronat, A. (2000) Carotenoid Biosynthesis during Tomato Fruit Development: Regulatory Role of 1-Deoxy-D-Xylulose 5-Phosphate Synthase. The Plant Journal, 22, 503-513. http://dx.doi.org/10.1046/j.1365-313x.2000.00764.X

[13] Banerjee, A., Wu, Y., Banerjee, R., Li, Y., Yan, H. and Sharkey, T.D. (2013) Feedback Inhibition of Deoxy-D-Xylulose-5-Phosphate Synthase Regulates the Methylerythritol 4-Phosphate Pathway. The Journal of Biological Chemistry, 288, 16926-16936. http://dx.doi.org/10.1074/jbc.M113.464636

[14] Hoeffler J.F., Tritsch, D., Grossdemange-Billiard, C. and Rohmer, M. (2002). Isoprenoid Biosynthesis via the Methylerythritol Phosphate Pathway: Mechanistic Investigations on the 1-Deoxy-D-Xylulose 5-Phosphate Reductoisomerase. European Journal of Biochemistry, 269, 4446-4457. http://dx.doi.org/10.1046/j.1432-1033.2002.03150.x

[15] Querol, J., Rodriguez-Concepcion, M., Boronat, A. and Imperial, S. (2001) Essential Role of Residue H49 for Activity of Escherichia coli 1-Deoxy-D-Xylulose 5-Phosphate Synthase, the Enzyme Catalyzing the First Step of the 2-C-Methyl-D-Erythritol 4-Phosphate Pathway for Isoprenoid Synthesis. Biochemical and Biophysical Research Communications, 289, 155-160. http://dx.doi.org/10.1006/bbrc.2001.5957

[16] Altincicek, B., Hintz, M., Sanderbrand, S., Wiesner, J., Beck, E. and Jomaa, H. (2000) Tools for Discovery of Inhibitors of the 1-Deoxy-D-Xylulose 5-Phosphate (DXP) Synthase and DXP Reductoisomerase: An Approach with Enzymes from the Pathogenic Bacterium Pseudomonas aeruginosa. FEMS Microbiology Letters, 190, 329-333.

[17] Xiang, S., Usunow, G., Lange, G., Busch, M. and Tong, L. (2007) Crystal Structure of 1-Deoxy-D-Xylulose 5-Phosphate Synthase, a Crucial Enzyme for Isoprenoids Biosynthesis. The Journal of Biological Chemistry, 282, 2676-2682. http://dx.doi.org/10.1074/jbc.M610235200

[18] Wikner, C., Meshalkina, L., Nilsson, U., Nikkola, M., Lindqvist, Y., Sundstrom, M. and Schneider, G. (1994) Analysis of an Invariant Cofactor-Protein Interaction in Thiamin Diphosphate-Dependent Enzymes by Site-Directed Mutagenesis. Glutamic Acid 418 in Transketolase Is Essential for Catalysis. The Journal of Biological Chemistry, 269, 3214432150.

[19] Wikner, C., Nilsson, U., Meshalkina, L., Udekwu, C., Lindqvist, Y., Schneider, G. and Schneider, G. (1997) Identification of Catalytically Important Residues in Yeast Transketolase. Biochemistry, 36, 15643-15649. http://dx.doi.org/10.1021/bi971606b

[20] Singleton, C.K., Wang, J.J., Shan, L. and Martin, P.R. (1996) Conserved Residues Are Functionally Distinct within Transketolases of Different Species. Biochemistry, 35, 15865-15869. http://dx.doi.org/10.1021/bi9616920

[21] Nilsson, U., Hecquet, L., Gefflaut, T., Guerard, C. and Schneider, G. (1998) Asp477 Is a Determinant of the Enantioselectivity in Yeast Transketolase. FEBS Letters, 424, 49-52. http://dx.doi.org/10.1016/S0014-5793(98)00136-7

[22] Matsue, Y., Mizuno, H., Tomita, T., Asami, T., Makoto Nishiyama, M. and Kuzuyama, T. (2010) The Herbicide Ketoclomazone Inhibits 1-Deoxy-D-Xylulose 5-Phosphate Synthase in the 2-C-Methyl-D-Erythritol 4-Phosphate Pathway and Shows Antibacterial Activity against Haemophilus Influenza. The Journal of Antibiotics, 63, 583-588. http://dx.doi.org/10.1038/ja.2010.100

[23] Handa, S., Ramamoorthy, D., Spradling, T.J., Guida, W.C., Adams, J.H., Bendinskas, K.G. and Merkler, D.J. (2013) Production of Recombinant 1-Deoxy-D-Xylulose-5-Phosphate Synthase from Plasmodium vivax in Escherichia coli. FEBS Open BIO, 3, 124-129. http://dx.doi.org/10.1016/j.fob.2013.01.007 